\title{
MAXIMALITY OF ORDERS IN NUMBER FIELDS
}

\author{
BEATA ROTHKEGEL \\ Institute of Mathematics, University of Silesia \\ Bankowa 14, 40-007 Katowice, Poland \\ ORCID:0000-0002-9953-5667_E-mail: beata.rothkegel@us.edu.pl
}

Abstract. In the paper, we formulate equivalent conditions for an order in a number field to be maximal.

1. Introduction. Let $K$ be a number field and let $R_{K}$ be the ring of integers of $K$. An order in $K$ is a subring $\mathcal{O}$ of $R_{K}$ which contains an integral basis of length $[K: \mathbb{Q}]$. The $\operatorname{ring} R_{K}$ is an order in $K$ and it is called the maximal order (cf. [N], Chapter I, (12.1) Definition]).

According to [N, Chapter I, (12.2) Proposition], every order in $K$ is a one-dimensional Noetherian domain with the field of fractions $K$.

The following ideal of $R_{K}$ is associated with an order $\mathcal{O}$ :

$$
\mathfrak{f}=\left\{a \in R_{K}: a R_{K} \subseteq \mathcal{O}\right\} .
$$

This ideal is called the conductor of $\mathcal{O}$. It is nonzero and it is the greatest ideal of $R_{K}$ lying in $\mathcal{O}$ (cf. [N, p. 79]).

ExAmple 1.1. Let $K=\mathbb{Q}(\sqrt{d})$, where $d$ is a square-free integer. Then

$$
R_{K}= \begin{cases}\mathbb{Z}[\sqrt{d}] & \text { when } d \not \equiv 1(\bmod 4), \\ \mathbb{Z}\left[\frac{1+\sqrt{d}}{2}\right] & \text { when } d \equiv 1(\bmod 4) .\end{cases}
$$

Moreover,

$$
\mathcal{O}= \begin{cases}\mathbb{Z}[f \sqrt{d}] & \text { when } d \not \equiv 1(\bmod 4) \\ \mathbb{Z}\left[f \frac{1+\sqrt{d}}{2}\right] & \text { when } d \equiv 1(\bmod 4)\end{cases}
$$

2010 Mathematics Subject Classification: Primary 16H15; Secondary 11R99.

Key words and phrases: order, Picard group, divisor, Witt ring.

The paper is in final form and no version of it will be published elsewhere. 
for some $f \in \mathbb{N}$ (cf. [BS, p. 151]). The conductor of $\mathcal{O}$ is a principal ideal generated by $f$; $\mathfrak{f}=f R_{K}$.

The question is as follows: when is $\mathcal{O}$ the maximal order? Obviously, if it is integrally closed. Indeed, $\mathcal{O} \subseteq R_{K}$. Take $a \in R_{K}$. Then $a$ is integral over $\mathbb{Z}$, so it is integral over $\mathcal{O}$. But $\mathcal{O}$ is integrally closed, so $a \in \mathcal{O}$ and $R_{K} \subseteq \mathcal{O}$.

In the paper, we consider the maximality of $\mathcal{O}$ in another context. We formulate equivalent conditions for the maximality of $\mathcal{O}$ using some homomorphisms between objects related to orders. The paper contains natural conclusions from the results of [R2] for orders in number fields.

Let $K$ be a number field and let $\mathcal{O}$ be an order in $K$. We write $\operatorname{Spec}(\mathcal{O}), \operatorname{Pic}(\mathcal{O})$ and cl $I$ for the maximal spectrum of $\mathcal{O}$, the Picard group of $\mathcal{O}$ and the class of an invertible fractional ideal $I$ of $\mathcal{O}$ in $\operatorname{Pic}(\mathcal{O})$, respectively.

The group $\operatorname{Pic}(\mathcal{O})$ is generated by all invertible ideals of $\mathcal{O}$ modulo the principal ideals $a \mathcal{O}, 0 \neq a \in K$ (cf. [N] Chapter I, (12.5) Definition]). From [N, Chapter I, (12.12) Theorem and p. 75], it follows that it is finite and if $\mathcal{O}=R_{K}$ is the maximal order, then $\operatorname{Pic}\left(R_{K}\right)$ is the ideal class group $\mathrm{Cl}_{K}$ of $K$. We write $h_{K}$ for the class number $\# \mathrm{Cl}_{K}$.

Throughout the paper, $U(P)$ and $U_{K}$ denote the group of invertible elements of a commutative ring $P$ and the group $U\left(R_{K}\right)$ of units of $K$, respectively.

2. Picard group and divisors of $\mathcal{O}$. Consider the natural homomorphism $\operatorname{Pic}(\mathcal{O}) \rightarrow$ $\mathrm{Cl}_{K}$ defined by

$$
\mathrm{cl} I \mapsto \mathrm{cl}\left(I R_{K}\right) \quad \text { for all } \mathrm{cl} I \in \operatorname{Pic}(\mathcal{O}) \text {. }
$$

We call it the Picard group homomorphism and by [ $[\overline{\mathrm{R} 2}$, Lemma 2.4] it is surjective.

Since the group $\operatorname{Pic}\left(R_{K}\right)$ is finite, from [R2, Theorem 4.1], the following fact follows.

THEOREM 2.1. Let $K$ be a number field and let $\mathcal{O}$ be an order in $K$. The following conditions are equivalent.

(1) $\mathcal{O}$ is the maximal order.

(2) The Picard group homomorphism is an isomorphism and $U_{K} \subseteq \mathcal{O}$.

(3) The Picard group homomorphism is injective and $U_{K} \subseteq \mathcal{O}$.

Corollary 2.2. Let $K$ be a number field and let $\mathcal{O}$ be an order in $K$. Then $\mathcal{O}$ is the maximal order if and only if

(1) $\# \operatorname{Pic}(\mathcal{O})=h_{k}$.

(2) $U_{K} \subseteq \mathcal{O}$.

ExAmple 2.3. Consider $K=\mathbb{Q}(\sqrt{d})$, where $d<0$ is a square-free integer and $d \equiv$ $5(\bmod 8)$. Moreover, let $\mathcal{O}=\mathbb{Z}\left[f \frac{1+\sqrt{d}}{2}\right]$ for some $f \in \mathbb{N}$.

Suppose $f \neq 1$. If $d=-3$, then $U_{K}=\left\{ \pm 1, \pm \frac{1 \pm \sqrt{-3}}{2}\right\} \nsubseteq \mathcal{O}$. Therefore we assume $d \neq-3$.

Now $U_{K}=\{ \pm 1\} \subseteq \mathcal{O}$. From [N, Chapter I, (12.12) Theorem], it follows that

$$
\# \operatorname{Pic}(\mathcal{O})=h_{K} \frac{\# U\left(R_{K} / \mathfrak{f}\right)}{\# U(\mathcal{O} / \mathfrak{f})} .
$$

We show that $\# \operatorname{Pic}(\mathcal{O}) \neq h_{K}$. 
Indeed, assume $f \neq 2$. Then $\sqrt{d} \notin \mathcal{O}$. If $\operatorname{gcd}(d, f)=1$, then there exist $x, y \in \mathbb{Z}$ such that $d x+f y=1$. Hence

$$
(\sqrt{d}+\mathfrak{f})(\sqrt{d} x+\mathfrak{f})=1+\mathfrak{f}
$$

and $\sqrt{d}+\mathfrak{f} \in U\left(R_{K} / \mathfrak{f}\right) \backslash U(\mathcal{O} / \mathfrak{f})$.

Let $p$ be a prime number such that $p \mid d$ and $p \mid f$. It is easy to observe that $1+\frac{f}{p} \sqrt{d} \notin \mathcal{O}$ and $\operatorname{gcd}\left(1-\frac{f^{2}}{p^{2}} d, f\right)=1$. Then $\left(1-\frac{f^{2}}{p^{2}} d\right) x+f y=1$ for some $x, y \in \mathbb{Z}$ and

$$
\left[\left(1+\frac{f}{p} \sqrt{d}\right)+\mathfrak{f}\right]\left[\left(1-\frac{f}{p} \sqrt{d}\right) x+\mathfrak{f}\right]=1+\mathfrak{f} .
$$

Moreover, $\left(1+\frac{f}{p} \sqrt{d}\right)+\mathfrak{f} \in U\left(R_{K} / \mathfrak{f}\right) \backslash U(\mathcal{O} / \mathfrak{f})$.

Assume $f=2$. Since $d \equiv 5(\bmod 8)$, the integer $\frac{1-d}{4}$ is odd and $\operatorname{gcd}\left(\frac{1-d}{4}, f\right)=1$. Similarly as above, there exist $x, y \in \mathbb{Z}$ such that $\frac{1-d}{4} x+f y=1$. Hence

$$
\left(\frac{1+\sqrt{d}}{2}+\mathfrak{f}\right)\left(\frac{1-\sqrt{d}}{2} x+\mathfrak{f}\right)=1+\mathfrak{f}
$$

and $\frac{1+\sqrt{d}}{2}+\mathfrak{f} \in U\left(R_{K} / \mathfrak{f}\right) \backslash U(\mathcal{O} / \mathfrak{f})$.

Finally, $\# U\left(R_{K} / \mathfrak{f}\right) \neq \# U(\mathcal{O} / \mathfrak{f})$, i.e. $\# \operatorname{Pic}(\mathcal{O}) \neq h_{K}$.

Some homomorphism between the Picard group and the Chow group is associated with the maximality of $\mathcal{O}$.

First, consider the group $C(\mathcal{O})$ of Cartier divisors and the group $\operatorname{Div}(\mathcal{O})$ of Weil divisors of $\mathcal{O}$. The first one is a multiplicative group generated by all invertible ideals of $\mathcal{O}$ (cf. [E, Corollary 11.7]) and the second one is a free abelian group generated by all maximal ideals of $\mathcal{O}$ (cf. [E, pp. 225, 259]).

Let $I \neq 0$ be an invertible ideal in $\mathcal{O}, \mathfrak{p} \in \operatorname{Spec}(\mathcal{O})$ be a maximal ideal and $I_{\mathfrak{p}}$ be the localization of $I$ at $\mathfrak{p}$. Moreover, let length $\left(\mathcal{O}_{\mathfrak{p}} / I_{\mathfrak{p}}\right)$ denotes the length of the ring $\mathcal{O}_{\mathfrak{p}} / I_{\mathfrak{p}}$.

From [E, Theorem 11.10 and its proof], it follows that length $\left(\mathcal{O}_{\mathfrak{p}} / I_{\mathfrak{p}}\right)<\infty$ and there is a group homomorphism $g: C(\mathcal{O}) \rightarrow \operatorname{Div}(\mathcal{O})$ defined by

$$
g(I)=\sum_{\mathfrak{p} \in \operatorname{Spec}(\mathcal{O})} \text { length }\left(\mathcal{O}_{\mathfrak{p}} / I_{\mathfrak{p}}\right) \cdot \mathfrak{p}
$$

for all invertible ideals $I$ in $\mathcal{O}$. We call it the length homomorphism.

In the case when $\mathcal{O}=R_{K}$ is the maximal order, the length homomorphism is injective (cf. E, Proposition 11.11]). Theorem 2.4 shows that the injectivity of $g$ is an equivalent condition for $\mathcal{O}$ to be maximal.

THEOREM 2.4. Let $K$ be a number field and let $\mathcal{O}$ be an order in $K$. The following conditions are equivalent.

(1) $\mathcal{O}$ is the maximal order.

(2) The length homomorphism is an isomorphism.

(3) The length homomorphism is injective.

Proof. See [R2, proof of Theorem 3.1]. 
EXAmple 2.5. Consider $K=\mathbb{Q}(\sqrt{-3})$ and $\mathcal{O}=\mathbb{Z}\left[f \frac{1+\sqrt{-3}}{2}\right]$ for some $1 \neq f \in \mathbb{N}$. Then $\pm \frac{1 \pm \sqrt{-3}}{2} \notin \mathcal{O}$, so $\frac{1 \pm \sqrt{-3}}{2} \mathcal{O} \neq \mathcal{O}$. Since $\frac{1 \pm \sqrt{-3}}{2} R_{K}=R_{K}$, by [R2, Lemma 3.1], $\frac{1 \pm \sqrt{-3}}{2} \mathcal{O} \in \operatorname{ker} g$. The length homomorphism is not injective.

Let Chow $(\mathcal{O})$ be the Chow group of $\mathcal{O}$. It is the group of Weil divisors of $\mathcal{O}$ modulo the principal divisors $g(a \mathcal{O}), 0 \neq a \in K$ (cf. [E, p. 260]). The length homomorphism $g$ induces a homomorphism $\bar{g}: \operatorname{Pic}(\mathcal{O}) \rightarrow \operatorname{Chow}(\mathcal{O})$.

Similarly as the length homomorphism, the homomorphism $\bar{g}: \mathrm{Cl}_{K} \rightarrow \operatorname{Chow}\left(R_{K}\right)$ is injective (cf. [E, Proposition 11.11]).

TheOREm 2.6. Let $K$ be a number field and let $\mathcal{O}$ be an order in $K$. The following conditions are equivalent.

(1) $\mathcal{O}$ is the maximal order.

(2) The homomorphism $\bar{g}: \operatorname{Pic}(\mathcal{O}) \rightarrow \operatorname{Chow}(\mathcal{O})$ is an isomorphism and $U_{K} \subseteq \mathcal{O}$.

(3) The homomorphism $\bar{g}: \operatorname{Pic}(\mathcal{O}) \rightarrow \operatorname{Chow}(\mathcal{O})$ is injective and $U_{K} \subseteq \mathcal{O}$.

Proof. See [R2, proof of Theorem 4.2].

It is easy to observe that the group of Cartier divisors of $R_{K}$ is the group of all fractional ideals of $K$. We write $C_{K}$ for it.

There is a natural homomorphism $C(\mathcal{O}) \rightarrow C_{K}$ defined by

$$
I \mapsto I R_{K} \quad \text { for all } I \in C(\mathcal{O}) .
$$

We call it the Cartier group homomorphism.

THEOREM 2.7. Let $K$ be a number field and let $\mathcal{O}$ be an order in $K$. The following conditions are equivalent.

(1) $\mathcal{O}$ is the maximal order.

(2) The Cartier group homomorphism is an isomorphism.

(3) The Cartier group homomorphism is injective.

Proof. See [R2, proof of Theorem 2.1].

ExAmple 2.8. Consider $K=\mathbb{Q}(\sqrt{-3})$ and $\mathcal{O}=\mathbb{Z}\left[f \frac{1+\sqrt{-3}}{2}\right]$ for some $f \in \mathbb{N}$. If $f \neq 1$, then $\frac{1 \pm \sqrt{-3}}{2} \mathcal{O} \in \operatorname{ker}\left(C(\mathcal{O}) \rightarrow C_{K}\right)$. The Cartier group homomorphism is not injective.

From Theorem 2.7 the corollary follows (see [ $\mathrm{R} 2$, Corollary 2.1]).

Corollary 2.9. Let $K$ be a number field and let $\mathcal{O}$ be an order in $K$. Then $\mathcal{O}$ is the maximal order if and only if

(1) $C(\mathcal{O})$ is a torsion-free group.

(2) $U_{K} \subseteq \mathcal{O}$.

3. Witt ring. There is a natural homomorphism $W \mathcal{O} \rightarrow W K$ between the Witt rings of $\mathcal{O}$ and $K$ defined in the following way. If $M$ is a finitely generated projective $\mathcal{O}$-module, $\alpha: M \times M \rightarrow \mathcal{O}$ is a nonsingular bilinear form on $M$ and $\langle(M, \alpha)\rangle \in W \mathcal{O}$ is the similarity class of the inner product space $(M, \alpha)$, then

$$
\langle(M, \alpha)\rangle \mapsto\langle(N, \beta)\rangle,
$$


where $N=K \otimes_{\mathcal{O}} M$ and $\beta: N \times N \rightarrow K$ is a nonsingular bilinear form on $N$ defined by

$$
\beta(a \otimes m, b \otimes n)=a b \alpha(m, n) \quad \text { for all } a, b \in K, m, n \in M .
$$

In the case when $\mathcal{O}=R_{K}$ is the maximal order, the natural homomorphism $W R_{K} \rightarrow$ $W K$ is injective (cf. [K, Satz 11.1.1]).

In [CS1, CS2, Ciemała and Szymiczek examined the kernel of $W \mathcal{O} \rightarrow W K$. They proved that if $\mathcal{O}$ is not maximal, then the kernel of $W \mathcal{O} \rightarrow W K$ is a nilideal. Moreover, they showed that for every order $\mathcal{O}=\mathbb{Z}[f i], f \neq 1$, in the field $K=\mathbb{Q}(i)$ the natural homomorphism is not injective. They formulated the conjecture that for a number field $K$ and an order $\mathcal{O}$ in $K$ the homomorphism $W \mathcal{O} \rightarrow W K$ is injective if and only if $\mathcal{O}$ is maximal. We know that it is not true. If $K=\mathbb{Q}(\sqrt{d}), d \not \equiv 1(\bmod 4), \mathcal{O}=\mathbb{Z}[f \sqrt{d}]$ is an order such that $2 \nmid f$ and the radical of $f$ divides $d$, then the natural homomorphism $W \mathcal{O} \rightarrow W K$ is injective (cf. [R1, Theorem 2.2]). Therefore the injectivity of the natural homomorphism is not a sufficient condition for an order to be maximal. In R2], we find equivalent conditions for the maximality of $\mathcal{O}$.

Theorem 3.1. Let $K$ be a number field and let $\mathcal{O}$ be an order in $K$. Then $\mathcal{O}$ is the maximal order if and only if

(1) The natural homomorphism $W \mathcal{O} \rightarrow W K$ is injective.

(2) The group $C(\mathcal{O})$ does not contain a nontrivial element of odd order.

(3) $U_{K} \subseteq \mathcal{O}$.

Proof. See [R2, proof of Theorem 5.1].

Consider the subgroup $C^{2}(\mathcal{O})$ of squares of the group $C(\mathcal{O})$ and the restriction $\left.g\right|_{C^{2}(\mathcal{O})}$ of the length homomorphism $g: C(\mathcal{O}) \rightarrow \operatorname{Div}(\mathcal{O})$ to $C^{2}(\mathcal{O})$.

Corollary 3.2. Let $K$ be a number field and let $\mathcal{O}$ be an order in $K$. Then $\mathcal{O}$ is the maximal order if and only if

(1) The natural homomorphism $W \mathcal{O} \rightarrow W K$ is injective.

(2) The homomorphism $\left.g\right|_{C^{2}(\mathcal{O})}$ is injective.

(3) $U_{K} \subseteq \mathcal{O}$.

Proof. See [R2, proof of Corollary 5.1].

\section{References}

[BS] Z. Borevich, I. Shafarevich, Number Theory, Nauka, Moscow, 1985 (in Russian).

[CS1] M. Ciemała, K. Szymiczek, On natural homomorphisms of Witt rings, Proc. Amer. Math. Soc. 133 (2005), 2519-2523.

[CS2] M. Ciemała, K. Szymiczek, On injectivity of natural homomorphisms of Witt rings, Ann. Math. Sil. 21 (2007), 15-30.

[E] D. Eisenbud, Commutative Algebra. With a View Toward Algebraic Geometry, Grad. Texts in Math. 150, Springer, New York, 1999.

[K] M. Knebusch, Grothendieck- und Wittringe von nichtausgearteten symmetrischen Bilinearformen, S.-B. Heidelberger Akad. Wiss. Math.-Natur. Kl. 1969/1970, 93-157. 
[N] J. Neukirch, Algebraic Number Theory, Grundlehren Math. Wiss. 322, Springer, Berlin, 1999.

[R1] B. Rothkegel, Witt functor of a quadratic order, Math. Slovaca 68 (2018), 1339-1342.

[R2] B. Rothkegel, Maximality of orders in Dedekind domains, J. Algebra Appl. 19 (2020), 2050125. 